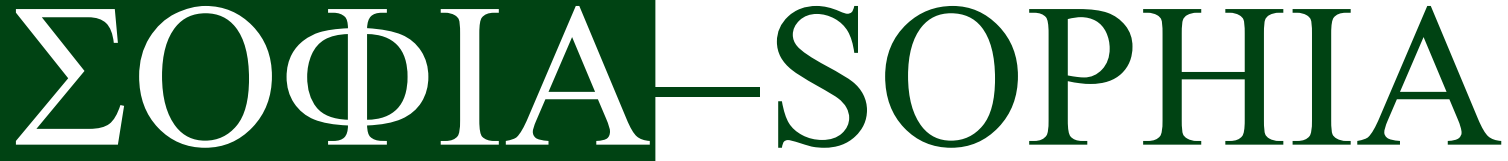

DOI: http://dx.doi.org/10.18634/sophiaj.14v.1i.824

\section{Escuela secundaria: currículo, saberes y prácticas en contexto*}

\section{High school: curriculum, knowledge and practices in context}

Ensino médio: currículo, conhecimento e práticas

em contexto

\author{
Horacio Ademar Ferreyra**
}

Información del artículo

Recibido: agosto de 2017 Revisado: octubre de 2017

Aceptado: enero de 2018

\section{Cómo citar:}

Ferreyra, H.A., (2018) Escuela secundaia: currículo, saberes y prácticas en contexto. Sophia 14 (1), 1-10.

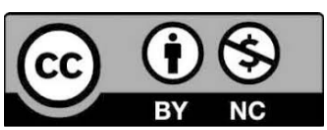

*En este artículo se presentan reflexiones sobre el currículo, saberes y prácticas en la escuela secundaria posible desde una perspectiva situada -conceptualizaciones, tensiones, problemáticas y propuestas-, como resultado del proceso de investigación desarrollado por el Equipo de Investigación de Educación de adolescentes y jóvenes de la Facultad de Educación de la Universidad Católica de Córdoba -Unidad asociada Conicet- (2014-2015) con la intencionalidad de contribuir con el debate educativo actual en torno a la temática.

**Doctor en Educación. Docente e investigador en la Universidad Católica de Córdoba (UCC) y Universidad Nacional de Villa María (UNVM) y Director del Equipo de Investigación de Educación de Adolescentes y Jóvenes (UCC, Unidad Asociada al Conicet (Consejo Nacional de Investigaciones Científicas y Técnicas de la República Argentina). Dirección electrónica hferreyra@, coopmorteros.com.ar. 


\title{
Resumen
}

En el marco de ejes y dimensiones que han permitido delinear una escuela posible, este artículo ahonda en el componente del currículo, articulándolo con las prácticas docentes y con los saberes pautados en el diseño oficial. Asimismo, caracteriza la dialéctica entre lo prescripto y lo habilitado en materia de contenidos a enseñar y a aprender, y las tensiones entre la selección, la organización y la secuenciación de contenidos y de aprendizajes. Finalmente, formula algunas propuestas para la conformación de un currículo posible.

Palabras clave: Currículo, educación, escuela secundaria, gestión curricular.

\begin{abstract}
Within the framework of axes and dimensions that have allowed to delineate a possible school, this article delves into the component of the curriculum, articulating it with the teaching practices and with the knowledge based on the official design. Likewise, it characterizes the dialectic between what is prescribed and what is enabled in terms of content to teach and to learn, and the tensions between the selection, organization and sequencing of contents and learning. Finally, it formulates some proposals for the formation of a possible curriculum.
\end{abstract}

Keywords: secondary school, curriculum, curricular management.

\section{Resumo}

Dentro do arcabouço de eixos e dimensões que permitiram delinear uma possível escola, este artigo investiga o componente do currículo, articulando-o com as práticas de ensino e com o conhecimento pautado no desenho oficial. Também caracteriza a dialética entre o que é prescrito e o que é habilitado em termos de conteúdo a ser ensinado e aprendido, e as tensões entre seleção, organização e sequenciamento de conteúdo e aprendizado. Finalmente, ele formula algumas propostas para a conformação de um currículo possível.

Palavras-chave: Currículo, educação, ensino médio, gestão curricular.

\section{Introducción}

\section{Consideraciones generales}

El currículo supone el análisis y la integración de componentes culturales, políticos, económicos, sociales y técnicos en una propuesta educativa que refleja, entre otras cuestiones, el tipo de sociedad que se aspira a construir (Gimeno Sacristán, 2010). Reconociendo el carácter multidimensional que es propio del currículo, Braslavsky (2002) lo conceptualiza como: "Un contrato denso y flexible entre la política, la sociedad y los docentes" (p.10) que por un lado prescribe y por otro, de manera simultánea, habilita cambios; así, compatibiliza y combina densidad y flexibilidad:
- Prescripciones generales que tienden a respaldar los procesos de implementación (densidad) y

- oportunidades claras y concretas de elegir y efectivizar opciones en el contexto de las instituciones educativas (flexibilidad).

El currículo es a la vez un proyecto político-pedagógico, por lo tanto, histórico, social, cultural y educativo, al que subyacen posiciones ideológicas, sociológicas, psicológicas, epistemológicas y pedagógicas ${ }^{1} \mathrm{y}$, por otro lado, las prácticas reales que se desarrollan desde este proyecto (Ferreyra, 2015). En esta línea, el diseño

1. En este principio se retoman y articulan conceptos de currículum desarrollados por diferentes autores en distintas obras, estudios e investigaciones; entre otros, véase: Schwab, 1974; Gimeno Sacristán, 1981; Stenhouse, 1984; Coll, 1986; Gimeno Sacristán, 1988; Elliot, 1990; Apple, 1996; Kemmis, 1998; Grundy, 1998. 
curricular es un componente de ese currículo y constituye para directivos, docentes, técnicos y supervisores un marco que regula la actuación profesional. Así, solo en la medida en que es entendido como herramienta de trabajo tiene la capacidad de generar, en cada contexto educativo, un proyecto de acción que hace posible articular las prescripciones y las prácticas en términos de construcción y enriquecimiento de las experiencias y de las trayectorias educativas de los estudiantes. Es decir, que no solo es currículo lo que se establece a través de documentos ${ }^{2}$, diseños, propuestas, sino también su desarrollo, aquellos saberes que efectivamente se enseñan y se aprenden en el aula y en la institución en su conjunto ${ }^{3}$.

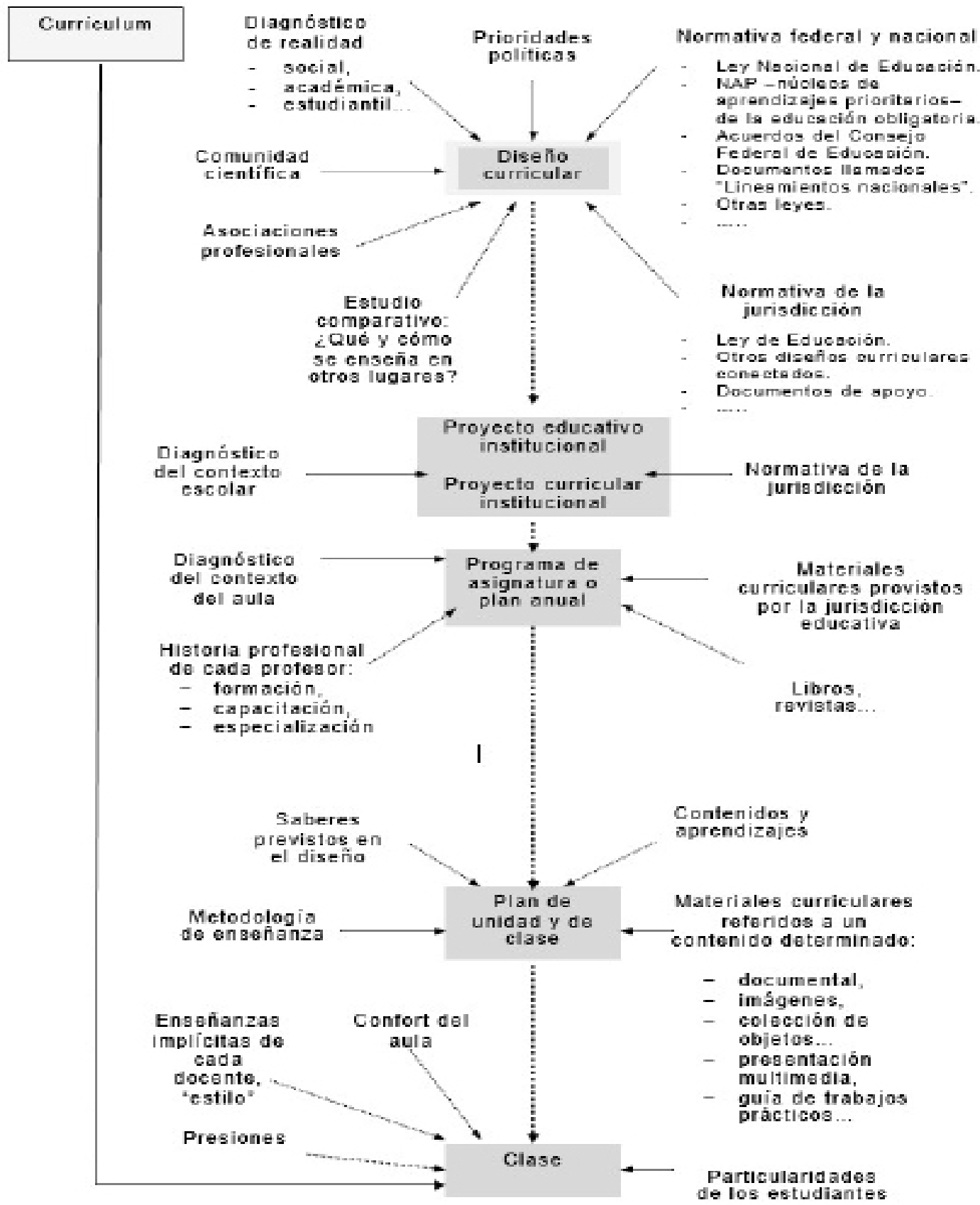

2. En la República Argentina existen NAP -Núcleos de Aprendizajes Prioritarios- acordados para todo el país; los diseños curriculares de cada jurisdicción educativa se ajustan a estos NAP. Por otra parte, en un nivel más contextualizado de concreción curricular, cada institución desarrolla el proyecto de la escuela -en algunas provincias /jurisdicciones educativas se lo denomina PEI- proyecto educativo institucional, con sus prioridades y sus distinciones; como componente de ese proyecto, los acuerdos que los profesores realizan acerca de los contenidos y de cómo enseñarlos están especificados en el proyecto curricular institucional (PCI).

3. Para ampliar, véase: De Alba, 1995; Connell, 1997; Terigi, 1999; Angulo y Blanco, 2000; Demeuse y Strauven, 2006, entre otros. 
En consonancia con lo expuesto, cabe decir que analizar el currículo implica también considerar las prácticas a través de las cuales se ponen en marcha los procesos de enseñanza y aprendizaje y el impacto que ellas generan en los sujetos participantes (estudiantes, docentes, directivos, entre otros), en su contexto.

Siguiendo a Alterman (2009), concebimos al currículo como proyecto de selección cultural de los saberes legitimados socialmente, un dispositivo de formación de los sujetos y un dispositivo de regulación de prácticas en contexto.

En esta idea amplia de currículo destacamos dos componentes centrales:

- Saberes: enunciados proposicionales (Cols, 2011) que expresan representaciones respecto de distintos campos de la realidad y que "son objeto de reconocimiento y control social, y son considerados como susceptibles de ser investidos en actividades de pensamiento, comunicación o transformación del entorno" ( $p .75)$.

- Contexto: Abarca los espacios y los vínculos de convivencia que en ellos se establecen a modo de entramado que incide y condiciona las relaciones, los roles y todos los procesos que se despliegan en la escuela.

\section{Currículo y educación secundaria: tensiones}

En relación con la educación secundaria, podemos destacar, en primer término, aquellas líneas de investigación y reflexión que ponen en relación cuestiones referidas a la organización curricular y pedagógica de la escuela secundaria con el impacto que genera en la inclusión y permanencia de los sujetos en el nivel. Las rescatamos como valiosas, porque como sostiene Zapiola (2011), la doble tensión entre obligatoriedad normativa ${ }^{4}$ e inclusión es el gran desafío para la educación secundaria argentina, en la que entran en tensión la antigua tradición de escuela elitista y la obligatoriedad de este nivel educativo para todos los jóvenes. Desigualdad social e inequidad del sistema parecen encontrarse con diseños escolares no siempre adecuados a la diversidad que puebla las escuelas secundarias argentinas, que por cierto siempre

\footnotetext{
4. Para una mejor comprensión del tema en el Documento preliminar para la discusión sobre la Educación Secundaria en Argentina (Consejo Federal de Educación de la República Argentina, 2008) se expresa: “... una de las metas más ambiciosas de la Ley de Educación Nacional es la extensión de la obligatoriedad para el nivel secundario. Alcanzarla constituye un imperativo para el Estado, para la sociedad en su conjunto y para los ciudadanos en particular. Estado, sociedad y ciudadanos se deben obligar a generar las condiciones de universalidad para que todas y todos los jóvenes puedan ingresar, permanecer y egresar de la escuela secundaria" (p.3).
}

estuvo presente, con diferentes matices, en las escuelas y en las aulas, pero que hasta pocas décadas atrás quedaba invisibilizada en una escuela atravesada por la homogeneidad.

La llegada a la Educación Secundaria de jóvenes de diversos sectores sociales y de distintas culturas, como dicen Jacinto y Terigi (2007):

Desestabilizó los acuerdos y los intereses previos. El nivel educativo está acogiendo a nuevos contingentes de alumnos que ya no adoptan las actitudes escolares y las motivaciones previstas. La escuela tiene que administrar a grupos heterogéneos y no le alcanza el desempeño de su rol tradicional (...). En este marco los trabajos especializados coinciden desde hace años en plantear la necesidad de reformular la selección curricular (pp. 38-39).

Jacinto y Terigi (2007) también coinciden en caracterizar al currículo de la escuela secundaria como significativamente clasificador, a partir de tres características que le son inherentes:

- Construcción de conocimientos disciplinares con marcadas delimitaciones entre campos,

- docentes seleccionados a partir de criterios clasificatorios y

- promoción de un conjunto de asignaturas troncales previstas en la mayoría de los currículos.

Estas características se mantuvieron estables durante todo el siglo XX y algunos intentos de compilación por áreas ${ }^{5}$ no han alterado la pauta clasificatoria.

En la misma dirección, Terigi (2008) señala que en la escuela secundaria se ha conformado un trípode de hierro, donde se forja la trama que enlaza un currículo clasificado y delimitado, en correlación con la designación de profesores por especialidad, en el marco de un trabajo docente por horas de clases en un currículo mosaico. También Feldman (2009) recurre a una metáfora para dar cuenta de una tríada distintiva del dispositivo pedagógico de la escuela secundaria: la "trinidad" conformada por el plan de estudios, el horario escolar y la división o sección: "Clasificación

\footnotetext{
5. En este sentido, basta recordar algunos proyectos y programas, tal el caso de $E s$ cuela para Jóvenes, programa impulsado por el Ministerio de Educación de la Nación en 2000 (Gobierno de Córdoba, 2010) para reformar la Educación Secundaria en la Argentina. Entre otras estrategias, y en búsqueda de una reorganización curricular en las escuelas, propuso una reducción de la cantidad de espacios curriculares de cursado simultáneo durante un año escolar y facilitar la concentración horaria de los profesores. Para ello se readecuaron los planes de estudio de las instituciones en torno a algunas áreas curriculares. Para ampliar información sobre resultados de implementación de este programa, véase, asimismo: Iaies, Delich y Gamallo, 2002.
} 
tripartita del conocimiento, el tiempo y el agrupamiento de alumnos (...) escenario básico de la vida escolar" (p. 63). A esto se suma el supuesto de la presencialidad (Terigi, 2010) por lo que: "Tenemos dificultades para dar la respuesta pedagógica adecuada a estas formas de presencia en la escuela que no cumplimentan con aquella expectativa que tenemos que es la de todos los días, todo el tiempo". (p. 11).

Las fracturas en la trayectoria escolar de los estudiantes también se asocian a saberes de escasa relevancia social, poco significativos para ellos, y que denotan todavía una débil presencia de temáticas emergentes y/o transversales (sexualidad, adicciones, convivencia, ambiente, cultura vial, cooperativismo y mutualismo, entre otras), de interés para los adolescentes y jóvenes, así como la gradual incorporación de las Tecnologías de la Información y la Comunicación (TIC) en los procesos de enseñanza y de aprendizaje (Unicef, 2015).

Con esto se corre el riesgo de que el currículo aparezca ajeno y distante para los estudiantes, sin sentido para sus vidas y con escasas posibilidades de generar motivación, con el agravante de que un currículo de estas características puede volverse aún más extraño para los estudiantes de sectores vulnerables que no cuentan en sus contextos con incentivos que contribuyan a encontrarle sentido a los aprendizajes escolares.

En esta línea, Montes (2008) sostiene que la posibilidad de avanzar hacia el cumplimiento efectivo de la universalización del nivel secundario como una condición básica de la inclusión social y de la concreción de los proyectos de vida de los jóvenes, obliga a interrogarnos: "Acerca de la factibilidad que tiene la educación secundaria de extenderse conservando su matriz original, configurada disciplinariamente en la Modernidad con trayectorias ideales previstas por nivel y año, bajo formatos uniformes y estandarizados" (p. 52 ). En una dirección semejante se pronuncia Romero (2009) cuando afirma que la posibilidad de alcanzar efectivamente la universalización de la educación secundaria se juega en la ruptura con "El formato academicista que primó durante décadas y sustituirlo por formas flexibles y creativas capaces de reconfigurar el conocimiento científico en conocimiento a aprender de la complejidad social, política e intelectual"'(p. 9); un conocimiento que se produce, se interroga y se habilita, agregaríamos acordando con Montes (2008). Un cambio de sentido en las formas de organizar la vida escolar permite potenciar los aprendizajes a partir de un fortalecimiento del vínculo del estudiante con el docente y con los saberes, en su contexto, para transformar estos últimos en conocimiento.
Dussel 2010 señalaba que los intentos por desarrollar estrategias de readecuación de la organización institucional y pedagógica de la escuela secundaria para incorporar contenidos nuevos, de relevancia social y más próximos a las problemáticas de los jóvenes, o nuevos actores que acompañen las trayectorias de los estudiantes (tutores, coordinadores pedagógicos, facilitadores TIC, entre otros) suelen estar circunscriptos a proyectos piloto o a programas predominantemente acotados a poblaciones en situaciones de vulnerabilidad socioeducativa. Hoy podemos decir que estas preocupaciones han comenzado a ser abordadas con intencionalidad de universalizar los procesos de mejora y que, a partir de la implementación de los Planes de Mejora de cada institución escolar de educación secundaria, estos nuevos actores o funciones están presentes en la mayoría de las escuelas secundarias del país.

Como síntesis de cierre de este apartado, podemos afirmar que la consideración del componente curricular en el marco de la escuela secundaria posible implica atender a los criterios de:
- $\quad$ Selección de aprendizajes y contenidos (saberes legítimos),
- $\quad$ organización (clasificación de esos aprendizajes $\mathrm{y}$ contenidos) y
- $\quad$ secuenciación y teorías de la enseñanza y del aprendizaje que dan sentido a estas opciones, en ocasiones en tensión.

\section{Definiendo algunas problemáticas}

Planteadas algunas directrices generales que permiten leer globalmente el campo de tensiones de la educación secundaria en la dimensión del currículo, los saberes y las prácticas, formulamos a continuación algunos problemas construidos en diálogo con investigaciones y producción ensayística de la última década:

a. Si bien los diseños y propuestas jurisdiccionales ponen en evidencia definiciones orientadas a superar la fragmentación y desarticulación curricular, y prescriben diversidad de formatos pedagógicos y alternativas para la enseñanza, en el proyecto curricular institucional persisten, en algunos casos, diseños formales que prevén modalidades organizativas homogéneas, con escaso margen para la variabilidad curricular.

$b$. Descontextualización del currículo en relación con la diversidad geográfica de nuestro territorio nacional. 
Atender a especificidades tales como las que son propias de la escolaridad en contextos de ruralidad resulta insuficiente; como señala Terigi (2012) es necesario el reconocimiento de la propia especificidad de los centros urbanos.

c. En la programación, selección, organización y secuenciación de contenidos que realiza cada docente, así como en el diseño de actividades previstas para su desarrollo, suelen aparecer sesgos de representaciones sociales y escolares tradicionales acerca de las evidencias de aprendizaje; por ejemplo: es frecuente que los educadores expresen que se está aprendiendo más si "se ven muchos temas" y los estudiantes "escriben todo en sus carpetas" (la oralidad se percibe, muchas veces, como "pérdida de tiempo", "no estar haciendo nada", "desorganización de la clase"); si la actividad es grupal, se sostiene que los estudiantes "aprenden poco" y que se dificulta la evaluación; se dice que es necesario avanzar desde "lo más simple" a "lo más complejo", lo que suele dar lugar a secuencias de aprendizaje tergiversadas que sustraen la posibilidad de que los jóvenes aborden la complejidad y multidimensionalidad de conceptos, hechos, fenómenos, lenguajes, prácticas.

d. La fragmentación de los contenidos redunda, muchas veces, en una desnaturalización del objeto de enseñanza y aprendizaje. Si bien la matriz predominantemente disciplinar de la educación secundaria ha sido señalada como una de sus debilidades, nos parece pertinente recuperar algunos reparos a la posible simplificación de que pudiera ser objeto esta afirmación con el propósito de aportar a una más clara delimitación del problema. Como aporta Fumagalli (2000) "Equiparar «fragmentación curricular» con "organización de los contenidos de enseñanza en asignaturas, disciplinas o materias» resulta una simplificación que no sólo no ayuda a pensar la complejidad del problema, sino que desplaza el foco del análisis" (p.78). De lo que se trata es de pensar la fragmentación como problema, en términos de construcción de saberes y advertir cómo una organización fragmentaria afecta los procesos de enseñanza y aprendizaje en el aula e impacta sobre los sujetos, restando oportunidades para la construcción de esquemas de conocimientos amplios y profundos.

e. Manifestación de la brecha existente entre generaciones en el acceso y el uso que se hace de las nuevas tecnologías en las instituciones educativas, y dificultades para operar las transformaciones que las TIC imponen a la organización del tiempo y del espacio, a las dinámicas y modos de gestión de las clases, a las maneras de apropiarse de los saberes y dialogar con ellos, a las relaciones de autoridad y jerarquía epistémica en el aula (en relación con las TIC, muchas veces son los estudiantes los que detentan el conocimiento, quienes saben más que el profesor). En este sentido, queda pendiente seguir fortaleciendo el desarrollo de nuevas prácticas pedagógicas con TIC para que la escuela se posicione efectivamente como un ámbito de aprendizajes personalmente significativos y socialmente relevantes (Dussel y Quevedo, 2010; Lugo y Kelly, 2011).

$f$. Estrategias didácticas en las que predomina la lógica de la linealidad y el orden secuencial se enfrentan con modos de aprender desde la lógica iconográfica e hipertextual, confrontación que marca una distancia cultural entre profesores y estudiantes. A esto se suman formas de evaluación muchas veces centradas más en el control y la medición que en la comprensión.

g. Dificultades para lograr la articulación intra (entre ciclos) e interinstitucional tanto con el nivel precedente (primario) como con el siguiente (superior), lo cual problematiza no solo los procesos implicados en los momentos de transición, sino también los de continuidad de estudios en el nivel subsiguiente. Entre los diversos factores incidentes ocupa un lugar fundamental el hecho de que cada tramo de escolaridad se ha desarrollado históricamente dando lugar a tradiciones propias y subculturas académicas diferenciadas. La escasa articulación se manifiesta también en la relación escuela-trabajo.

$h$. El sistema de acreditación del nivel secundario argentino presenta -aunque se observan avancesbastante rigidez asociada con formas establecidas en la modernidad y que actúan debilitando, en muchas oportunidades, las posibilidades de cambios estructurales en la organización de tiempos y espacios de la institución, que podrían acompañar las trayectorias de los estudiantes desde sus particularidades.

\section{Propuestas posibles}

La mera enunciación de problemas de la escuela secundaria nos llevaría a quedar atrapados en la imposibilidad de pensar cambios profundos y sobre todo que se puedan llevar a cabo a mediano y largo plazo. Para revertir este riesgo, presentamos algunas propuestas que surgen de lo investigado hasta el momento:

a. Profundizar los cambios para la educación secundaria desde una referencia política amplia e integradora, que 
contemple los planos político, cultural, económico y social, con lineamientos y principios que construyan una trama educativa. Estos cambios planteados por la macropolítica desde los que cada provincia consolida su proyecto, van a concretizarse en las micropolíticas institucionales esbozadas por cada escuela, en sus itinerarios de transformación.

b. Fortalecer las propuestas curriculares en las instituciones de formación de docentes de distintos campos disciplinares de la escuela secundaria. El objetivo es producir una transformación estructural de largo alcance, a los efectos de no caer en una formación descontextualizada.

c. Profundizar la formación de los equipos institucionales de conducción para que continúen movilizando las transformaciones del nivel educativo (macro) e intrainstitucionales (micro) que las políticas educativas proponen para la escuela secundaria. Ya no se trata de un modelo basado en el prototipo unipersonal, sino de equipos colegiados que asumen acciones en procesos compartidos y colaborativos, generadores de una sinergia capaz de liderar los procesos transformadores.

d. Dar continuidad a los procesos de revisión y construcción de un currículo flexible, articulado (horizontal y verticalmente), regionalizado y contextualizado (legitimándolo en las características de la vida social fuera de la escuela), desde una perspectiva participativa de todos los sujetos del sistema educativo. En la tensión entre lo común y lo diverso, entonces, cabe tener presente la advertencia de Terigi (2009):

Que esa formación compartida no arrase con las singularidades y la cultura local, ni codifique como única cultura autorizada la de sectores específicos de la población; por el contrario, que promueva en todos una comprensión de la cultura y de los intereses de los otros (pp. 10-11).

Esto demanda dar cabida a nuevas modalidades organizativas que amplíen y enriquezcan las oportunidades de aprendizaje que se ofrecen a los jóvenes; iniciar y sostener acciones graduales, propone Feldman (2009): "Que debiliten la fuerza que proyecta la tríada [plan de estudios, horario escolar y la división o sección] y abrir espacios para mejorar la experiencia escolar" (p. 71). Para ello, es necesario elaborar y/o fortalecer propuestas pedagógicas que contemplen otros tiempos (extensivos e intensivos), espacios (escolares y extraescolares, presenciales o no), estrategias y actividades (instancias de trabajo compartido entre espacios curriculares, alternancias, asignaturas electivas, pasantías y otras prácticas educativas en el contexto, ferias y muestras abiertas a la comunidad, entre otras posibilidades).

e. Fortalecer los procesos de selección, organización y secuenciación de aprendizajes significativos y relevantes, promoviendo la integración de los saberes en torno a ejes estructurantes, temas generadores, ámbitos de experiencia de los estudiantes (sexualidad, TIC, vida saludable ${ }^{6}$, etc.) entre otras posibilidades respecto de las cuales afirma Dussel (2008):

Hoy también corresponde plantearse qué otros mundos posibles pueden ofrecerse desde la escuela, en diálogo y en relación con el mundo en el que vivimos (...) en una época en la que todo es fluido e instantáneo, vale la pena insistir con el valor de la reflexión y de la inducción en saberes sistemáticos de las disciplinas, pero sin que se conviertan en cosas viejas y con sentido sólo al interior de la escuela, sino como saberes sometidos al tamiz de su valor social, de su capacidad para responder viejas y nuevas preguntas de las sociedades humanas. (p.15).

En esta línea, es necesario no solo incorporar y afianzar temáticas emergentes o transversales (adicciones, convivencia, cultura vial, ambiente, cooperativismo y mutualismo, etc.) en las propuestas curriculares sino también explorar nuevas formas de inclusión y abordaje en las prácticas de enseñanza que las contemplan. Además, se trata de ampliar la idea de alfabetizaciones básicas para incluir los saberes, interacciones y tecnologías que hoy son dominantes en nuestra sociedad, y formar a las nuevas generaciones para que puedan vincularse con ellas de maneras más creativas, más libres y más plurales. La clave es identificar qué es lo que efectivamente se tiene que enseñar y aprender en la escuela secundaria, en cada uno de sus ciclos y años, porque los estudiantes no han de aprenderlo en la vida o en los ciclos o años subsiguientes y, con esta vacancia, se afectaría seriamente no sólo su trayectoria escolar sino también su inclusión social. Esto nos ha de llevar a pensar gradualmente en cómo transformar un currículo centrado en contenidos en uno centrado en la adquisición y desarrollo de capacidades, lo cual supone pensar el currículo en términos de aprendizajes, precisando el alcance de los contenidos involucrados 6. Una temática transversal que hoy está siendo objeto de una consideración especial; se trata de incentivar, propiciar y enseñar a los estudiantes contenidos que se relacionan con los hábitos y costumbres para el logro de una vida saludable. Para ello, se pueden generar y planificar una serie de propuestas que permitan a los estudiantes no sólo conocer y valorar la importancia que tiene la buena alimentación, la higiene personal, la salud física y mental, entre otros factores, sino también crear espacios y situaciones escolares que permitan que estas cuestiones sean parte de la vida cotidiana. 
en ellos ${ }^{7}$.

f. Promover la planificación de diversos formatos curriculares: talleres, seminarios, proyectos, ateneos, observatorios, laboratorios, trabajo de campo, módulo, entre otros, de modo que su posterior implementación habilite diversas modalidades organizativas, distintos modos de relacionarse con el conocimiento y de hacerlo "circular" en las aulas, variedad de espacios y agrupamientos.

g. Continuar acompañando los procesos de implementación y desarrollo curricular a través de instancias de formación situada o centrada en la escuela, propiciando prácticas pedagógicas que contemplen la integración teoría-práctica de manera más clara y significativa, incluyendo también orientaciones para trabajar propuestas de articulación inter e intrainstitucional en contexto; el rescate de buenas prácticas en el desarrollo curricular también es otra alternativa posible. Asimismo, se promueve la estrategia de vinculación de la educación con el trabajo socialmente productivo, a través de instancias de participación de los estudiantes en su comunidad.

h. Resignificar las formas y sentidos de la evaluación mediante espacios de discusión en torno a los modelos de evaluación utilizados por los docentes, en los que se puedan reflexionar, definir y discutir criterios y formas de evaluación de los aprendizajes. Según Dubet (2010) dichos criterios y formas no necesariamente deben ser los mismos y comunes en todos los espacios curriculares o ante diversas propuestas; por el contrario -sostienedeben ser múltiples y diversos y no reducirse a la medición de conocimientos, sino que el bienestar de los estudiantes y sus capacidades sociales tendrían que ser criterios igualmente importantes de evaluación de una escuela democrática. También es necesario optimizar los mecanismos e instrumentos de socialización de los resultados de la evaluación con los estudiantes y con sus familias. Como punto nodal de todo proceso evaluativo, desplazar la consideración del aspecto negativo, de lo no logrado o no aprendido -es decir, del déficit- para focalizarla en los aspectos positivos: qué es lo que el estudiante efectivamente aprendió como base sobre la cual anclar los nuevos aprendizajes, a partir del reconocimiento de lo logrado.

$i$. Intensificar el trabajo en equipos interdisciplinarios e integrados de docentes que potencien los temas del currículo para mejorar y resignificar los aprendizajes (por ejemplo, con proyectos de trabajo en los que se

7. Esta perspectiva, distintiva de la enunciación de los NAP, ya es retomada en los diseños curriculares para la Educación Secundaria de la provincia de (Gobierno de Córdoba, 2011). vinculen dos o más disciplinas, asignaturas o espacios para el desarrollo de contenidos y para la evaluación).

j. Planificar, crear y sostener redes escolares que permitan trabajar coordinada y mancomunadamente con otras instituciones locales y extranjeras, potenciando de esta manera los vínculos formales y no formales tanto presenciales como virtuales- que permitan que las comunicaciones acerquen los tiempos y espacios y favorezcan el hacerse parte tanto de la comunidad de pertenencia como del mundo, resorte vital para disminuir la brecha que marca diferencias sociales, étnicas, religiosas y culturales.

$k$. Potenciar los espacios curriculares que se relacionan con la formación para la vida y el trabajo, haciendo que estas dos dimensiones crezcan en virtud de las características particulares de cada orientación de la educación secundaria, permitiendo, de este modo, que los estudiantes ingresen paulatinamente en la realidad del "afuera escolar" y se apropien de saberes imprescindibles para la construcción de un proyecto de vida que dé respuesta a sus necesidades e intereses personales como parte de la comunidad de pertenencia.

\section{Conclusiones}

Los procesos de transformación - y en particular, los de orden curricular- requieren ser pensados con una proyección a largo plazo que permita poner en discusión y reflexión componentes relacionados con lo histórico, lo social, lo cultural, lo económico y lo educativo. Proponer un currículo como proyecto político-pedagógico requiere decisiones políticas, pero no solo gubernamentales, sino desde todas las personas involucradas en las instituciones educativas: docentes, estudiantes y familias.

La educación plasmada en una propuesta curricular amplia involucra al colectivo social en su conjunto, ya que sus efectos inciden en todos los sujetos sociales que atraviesan las escuelas y la vida social. Para ello, es clave una formación profesional de los responsables -directivos y docentes- para una actualización y profundización que posibiliten una transformación estructural de la educación secundaria a largo plazo, no solo de contenidos, sino didáctica (recursos, propuesta metodológica y evaluativa).

Gran parte de las propuestas que aquí se han esbozado comienzan a ser transitadas por las distintas provincias/ jurisdicciones del país, lo que permite pensar que las transformaciones deseadas son posibles. Es necesario, 
entonces, afianzar políticas de largo plazo que potencien estos cambios.

\section{Referencias bibliográficas}

Alterman, N. (2009). Desarrollo curricular centrado en la escuela y en el aula. Fortalecimiento Pedagógico de las Escuelas del Programa Integral para la Igualdad Educativa. Buenos Aires: Ministerio de Educación de la Nación.

Angulo, J. y Blanco, N. (2000). Teoría y desarrollo del Currículo. Málaga, España: Aljibe.

Apple, M. (1996). El conocimiento oficial. La educación democrática en una era conservadora. Barcelona, España: Paidós.

Braslavsky, C. (2002). The New Century's Change: New Challenges and Curriculum Responses. [El cambio del nuevo siglo: nuevos desafíos y respuestas curriculares].

Conferencia Internacional del Council of Boards of School Education, República de la India. Nueva Delhi: COBSE.

Cols, E. (2011). Estilos de enseñanza. Sentidos personales y configuraciones de acción tras la semejanza de las palabras. Rosario, Argentina: Homo Sapiens.

Coll, C. (1986). Los niveles de concreción del diseño curricular. En Cuadernos de Pedagogía (139) pp. .23-30. Barcelona, España: Praxis.

Connell, R. (1997). Escuelas y Justicia social. Madrid: Morata.

Consejo Federal de Educación de la República Argentina (2008). Documento preliminar para la discusión sobre la Educación Secundaria. Buenos Aires: CFE.

DeAlba,A.(1995). Curriculum: crisis, mito y perspectiva. Buenos Aires: Miño y Dávila.

Demeuse, M y Strauven, C. (2006). Developper un curriculum d'enseignement ou de formation. Des options au pilotage. Introduction (pp 9-28). Bruxelles: De Boeck.

Dussel, I. (2008). Los debates del currículo en la actualidad: ¿cuáles son los saberes básicos que debería transmitir hoy la escuela? En El currículo. Documento del Programa de Capacitación Multimedial Explora. Buenos Aires: Ministerio de Educación, Ciencia y Tecnología de la Nación.

Dussel, I. (2010a). La escuela media argentina y los desafios de las metas 2021. Ponencia presentada en el V Foro Latinoamericano de Educación: Metas educativas 2021: propuestas iberoamericanas y análisis nacional. Buenos Aires: Santillana.

Dussel, I. y Quevedo L. (2010b). Educación y nuevas tecnologías: los desafios pedagógicos ante el mundo digital. Documento básico. VI Foro Latinoamericano de Educación. Buenos Aires: Santillana.

(2010). Declive de la institución escolar y conflictos de principios. Ponencia en Debates de Educación. Barcelona, España Fundación Jaume Bofill y Universitat Oberta de Catalunya.

Elliott, J. (1990). La investigación-acción en educación. Madrid: Morata.

Feldman, D. (2009). Currículo e inclusión educativa. Buenos Aires: Noveduc.

Ferreyra H. [et al.] (2015) Reflexiones sobre la calidad de la educación secundaria Argentina: la escuela posible como horizonte de expectativas. Córdoba, Argentina: Editorial UCC-Unicef Argentina.

Fumagalli, L. (2000). Alternativas para superar la fragmentación curricular en la educación secundaria a partir de la formación de los docentes. En Braslavsky, C., Dussel, I. y Scaliter, P. (eds.). Los formadores de jóvenes en América Latina. Desafios, experiencias $y$ propuestas. Informe final Seminario Internacional. Maldonado, Uruguay: UNESCO y Administración Nacional de Educación Pública del Uruguay.

Gimeno Sacristán, J. (1981). Teorías de la enseñanza y desarrollo del currículo. Madrid: Anaya.

(1988): El currículo: una reflexión sobre la práctica. Madrid: Morata. 
(2010). Saberes e incertidumbres sobre el currículo. Madrid: Morata.

Gobierno de Córdoba. Ministerio de Educación, Secretaría de Educación, Subsecretaría de Igualdad y Calidad Educativa. Área de Investigación Educativa (2010). Investigación I: Estudio de Impacto del Programa Escuela Para Jóvenes. Córdoba, Argentina: Autor.

Gobierno de Córdoba. Ministerio de Educación, Secretaría de Educación, Subsecretaría de Igualdad y Calidad Educativa (2011). Diseño curricular de la educación secundaria 2011-2020. Córdoba, Argentina: Autor.

Grundy, S. (1998) Producto o praxis del currículo. Madrid: Morata.

Iaies, G., Delich, A y Gamallo, G. (2002). Estudio de Evaluación: Implementación del Programa Escuela para Jóvenes en Córdoba. Buenos Aires: Centro de Estudios de Políticas Públicas.

Jacinto, C. y Terigi, F. (2007). ¿Qué hacer ante las desigualdades en la educación secundaria? Instituto Internacional de Planeamiento de la Educación, IIPE. Buenos Aires: Santillana.

Kemmis, S. (1998). El currículo: más allá de la teoría de la reproducción. Madrid: Morata.

Lugo, M. y Kelly, V. (2011). El modelo 1 a 1: un compromiso por la calidad y la igualdad educativas. La gestión de las TIC en la escuela secundaria: nuevos formatos institucionales. Buenos Aires: Ministerio de Educación de la Nación.

Montes, N. (2008). La educación secundaria en la región. Facultad Latinoamericana de Ciencias Sociales. Buenos Aires: Flacso.

Romero, C. (2009). Claves para mejorar la escuela secundaria. La gestión, la enseñanza y los nuevos actores. Buenos Aires: Noveduc.

Schwab, J. (1974). Un enfoque práctico para la planificación del currículo. Buenos Aires: El Ateneo.

Stenhouse, L. (1984). Investigación y desarrollo del currículo. Madrid: Morata.
Terigi, F. (1999). Currículo. Itinerarios para aprender un territorio. Buenos Aires: Santillana.

Terigi, F. (2008). Los cambios en el formato de la escuela secundaria: por qué son necesarios, por qué son tan difíciles. En Propuesta Educativa, 17 (29), 63-72. Facultad Latinoamericana de Ciencias Sociales. Buenos Aires.

(2009). Las trayectorias escolares. Del problema individual al desafio de politica educativa. Documento para el Proyecto Hemisférico: Elaboración de Políticas y Estrategias para la Prevención del Fracaso Escolar (OEA-AICD). Presentado en el Seminario Internacional "Dimensiones para el diseño de políticas de inclusión educativa", organizado por OEA-EUROsociAL sector Educación. Buenos Aires: Ministerio de Educación de la Nación.

-----(2010). Las cronologías de aprendizaje: un concepto para pensar las trayectorias escolares. Santa Rosa, Argentina: Ministerio de Cultura y Educación, Gobierno de La Pampa.

-(2012). Presentación. En Adolescentes y secundaria obligatoria Finalización de Estudios y Vuelta a la Escuela Conurbano. Buenos Aires: Unicef y UNGS, Universidad Nacional de General Sarmiento.

Unicef. (2015). Informe General. Resultados de la Encuesta Nacional sobre Integración de TIC en la Educación Básica Argentina. Programa TIC y Educación Básica. Buenos Aires: Fondo de las Naciones Unidas para la Infancia.

Zapiola, M. (2011). Infancias: politicas y saberes en Argentina y Brasil. Siglos XIX y XX. Buenos Aires: Teseo. 